International Journal of Pure and Applied Mathematics

Volume 87 No. 5 2013, 719-728

ISSN: 1311-8080 (printed version); ISSN: 1314-3395 (on-line version)

url: http://www.ijpam.eu

doi: http://dx.doi.org/10.12732/ijpam.v87i5.5

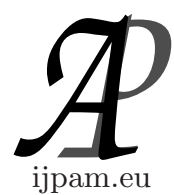

\title{
DETOUR DISTANCE PATTERN OF A GRAPH
}

\author{
Kumar Abhishek $^{1 \S}$, Ashwin Ganesan ${ }^{2}$ \\ ${ }^{1,2}$ Amrita School of Engineering \\ Amrita Vishwa Vidyapeetham \\ Coimbatore, 641 112, Tamil Nadu, INDIA
}

\begin{abstract}
For a simple connected graph $G=(V, E)$, let $M \subseteq V$ and $u \in V$. The $M$-detour distance pattern of $G$ is the set $f_{M}(u)=\{D(u, v): v \in M\}$. If $f_{M}$ is injective function, then the set $M$ is a detour distance pattern distinguishing set (or, $d d p d$ - set in short) of $G$. A graph $G$ is defined as detour distance pattern distinguishing (or, $d d p d-$ ) graph if it admits a $d d p d$-set. The objective of this article is to initiate the study of graphs that admit marker set $M$ for which $f_{M}$ is injective. This article establishes some general results on $d d p d$-graphs.
\end{abstract}

\section{AMS Subject Classification: $05 \mathrm{C} 22$}

Key Words: detour distance, detour distance pattern, detour distance pattern distinguishing set

\section{Introduction}

All the graphs considered here are finite, undirected, connected and simple. For standard graph theory terminology and notations not defined here, we refer [1].

Specifically, let $G=(V, E)$ be a graph with vertex set $V$ and edge set $E$. For arbitrary pair of vertices $u, v \in V$, the standard distance $d(u, v)$ is the length of the shortest $u-v$ path and the detour distance $D(u, v)$ is the length of the longest $u-v$ path between $u$ and $v$ in $G$. As with standard distance, detour distance is known to be a metric on the vertex set of any connected graph. Chartrand et.al, [2], were the first to introduce the notion of detour distances

Received: April 9, 2013

(C) 2013 Academic Publications, Ltd.

${ }^{\S}$ Correspondence author url: www.acadpubl.eu 
in graphs and was subsequently explored by many scholars. A variety of results pertaining to the detour distances in graphs are known to us. Before we proceed any further we recall the following results which we require subsequently.

Theorem 1. [2]

1. For any graph $G, D(u, v)=1$ if and only if $u v$ is a bridge in $G$.

2. For any graph $G, d(u, v)=D(u, v)$ for every pair of vertices $u$ and $v$ of $G$ if and only if $G$ is a tree.

The detour eccentricity $e_{D}(G)$ of a vertex $v$ is the detour distance from $v$ to a vertex farthest from $v$. The detour radius of $\operatorname{rad}_{D}(G)$ of a connected graph $G$ is the minimum detour eccentricity among the vertices of $G$ and the detour diameter $\operatorname{diam}_{D}(G)$ is the maximum detour eccentricity among the vertices of $G$. The detour center $C_{D}(G)$ of a graph $G$ is the subgraph of $G$ induced by those vertices of $G$ whose detour eccentricity is $\mathrm{rad}) d(G)$.

For a simple connected graph $G=(V, E)$, let $M \subseteq V(G)$ and $u \in V$. The $M$-detour distance pattern of $G$ is the set $f_{M}(u)=\{D(u, v): v \in M\}$. If $f_{M}$ is injective function, then the set $M$ is a detour distance pattern distinguishing set (or, $d d p d$ - set in short) of $G$. A graph $G$ is defined as detour distance pattern distinguishing (or, $d d p d$-) graph if it admits a $d d p d$-set.

It must be noted that for any graph $G$, with $|V| \geq 2, V$ is not a $d d p d$-set, since for any two vertices $u, v \in V$ with $D(u, v)=\operatorname{diam}_{D}(G)=D$ we have $f_{M}(u)=f_{M}(v)=\{0,1,2, \ldots, D\}$.

Further, for a given simple connected graph $G=(V, E)$, let $M \subseteq V(G)$ and $u \in V(G)$. The $M$-distance pattern of $G$ is the set $f_{M}(u)=\{d(u, v)$ : $v \in M\}$. If $f_{M}$ is injective function, then the set $M$ is a distance pattern distinguishing set (or, $d p d$ - set in short) of $G$. A graph $G$ is defined as distance pattern distinguishing (or, $d p d-$ ) graph if it admits a $d p d$-set. The notion of $d p d$-graph was introduced by Germina [3] which was subsequently investigated by many researchers since its inception in 2010 .

In this article, we initiate the study of $d d p d$-graph, by changing the standard distance $d(u, v)$ between two vertices $u$ and $v$ in a graph by its detour distance $D(u, v)$.

\section{A Few Facts Involving $d p d$-Graphs}

It was noted [3] that not every graph is a $d p d$-graph. For example, the complete graph $K_{n}, n \geq 3$ is does not possess a $d p d$-set, hence is not a $d p d$-graph. 
The following results have been established in the recent past in relation to $d p d$-graphs.

Theorem 2. [3] Let $G$ be any connected graph then, $G$ has a dpd-set of cardinality 1 if and only if it is a path.

Theorem 3. [4] Let $G$ be any connected graph. There exists no dpd-set of cardinality 2.

Theorem 4. [3] If $M$ be a dpd-set of $G$ then, the induced subgraph $\langle M\rangle$ is disconnected.

Theorem 5. [3] If $M$ be a dpd-set of $G$ then, any vertex of $G$ is adjacent to at most two pendant vertices. Further, if $G$ has a vertex with exactly two pendant vertices adjacent to it then, exactly one of them belongs to $M$.

The problem of characterizing $d p d$-graph remains to be an open problem since its inception. The following classes of graphs are known to be $d p d$-graph.

Theorem 6. [4] A cycle $C_{n}$ of order $n$ is a dpd-graph if and only if $n \geq 7$.

Theorem 7. [4] $K_{1, n}$ is a dpd-graph if and only if $n \leq 2$.

Theorem 8. [5] A double star $S_{m, n}$ is a dpd-graph if and only if $n, m \leq 2$.

Theorem 9. [5] A uniform binary tree is $T$ is a dpd-graph if and only if $O(T)=2^{m}-1$ where $m=1,2,3$.

Theorem 10. [5] k-uniform caterpillars are dpd-graphs if and only if $k=1$.

Theorem 11. [5] Every olive tree is a dpd-graph.

\section{3. $d d p d$-Graphs Versus $d p d$-Graphs}

The objective of this section is to do a comparative study of $d d p d-$ and $d p d-$ graphs. We begin this section with the following result which we state without a proof.

Proposition 12. If $u$ and $v$ are any two distinct vertices of a cycle $C_{n}$ of order $n$ then, $D(u, v)+d(u, v)=n$.

In view of Proposition 12 and Theorem 6 the notion of $d d p d$ - and $d p d$-graphs are equivalent for the case of cycles. Further in view of Theorem 1 the notion of $d d p d$ - and $d p d$-graphs are equivalent for the case of trees. Also, the notion of $d d p d$ - and $d p d$-graphs are equivalent for the case of complete graphs. With the 
above few instances of equivalence in the notion of $d d p d$ - and $d p d$-graphs the reader must not conclude that the two notions are equivalent in general. As the following example illustrates. Let $G$ be the graph as shown in the figure 1 .

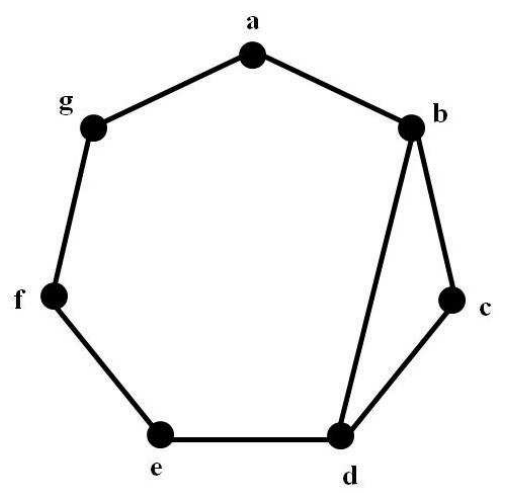

Figure 1: $G$

Consider the subset $M=\{a, b, d\} \subset V$. The $M$-detour distance pattern of the vertices of $G$ is the following:

$$
\begin{aligned}
f_{M}(a) & =\{0,4,6\} \\
f_{M}(b) & =\{0,5,6\} \\
f_{M}(c) & =\{5,6\} \\
f_{M}(d) & =\{0,4,5\} \\
f_{M}(e) & =\{4,6\} \\
f_{M}(f) & =\{4,5\} \\
f_{M}(g) & =\{4,5,6\} .
\end{aligned}
$$

Clearly $f_{M}$ is an injective function. Hence $M$ is a $d d p d$ - set of $G$. Note that the induced subgraph $\langle M\rangle$ is connected. Thus the induced subgraph of a $d d p d$-set need not be disconnected, whereas in view of Theorem 3 the induced subgraph $\langle M\rangle$ of a $d p d$-set has to be disconnected.

One can verify that the graph $G$ as shown in the figure 1 is not a $d p d$-graph.

Thus, $G$ is a $d d p d$-graph which is not $d p d$-graph. With the discussion of foregoing paragraph in mind it is quite natural to ask the following:

Open Problem 13. Characterize the ddpd-graphs for which the induced subgraph $\langle M\rangle$ is connected. 
Open Problem 14. Characterize the graphs which are both ddpd- and dpd-graph.

\section{Some General Results on DDPD-Graphs}

We begin this section by establishing some of the introductory results pertaining to $d d p d$-graphs.

Proposition 15. The set of all vertices in the detour diametral path of a graph $G$ cannot form a ddpd-set.

Proof. Let $P_{n}=u_{1}, u_{2}, u_{3}, \ldots, u_{n}$ be an arbitrary detour path. If possible let $M=\left\{u_{1}, u_{2}, u_{3}, \ldots, u_{n}\right\}$ be a $d d p d$-set of $G$. Then

$$
f_{M}\left(u_{1}\right)=\left\{0,1,2, \ldots, \operatorname{diam}_{D}(G)\right\}=f_{M}\left(u_{n}\right) .
$$

A contradiction to the fact that $M$ is a $d d p d$-set of $G$.

Using a similar argument the following is immediate

Proposition 16. The set of all vertices in the detour center of a graph $G$ cannot form a ddpd-set.

Our next result provides a necessary condition for a graph to admit $d d p d$-set of cardinality 3.

Proposition 17. If $M$ be a ddpd-set of $G$ such that $|M|=3$ then, the detour distances between any pair of vertices in $M$ must be distinct.

Proof. Assume to the contrary, let $M=\left\{v_{1}, v_{2}, v_{3}\right\}$ be a ddpd -set of $G$.

Case 1: $D\left(v_{1}, v_{2}\right)=D\left(v_{2}, v_{3}\right)=D\left(v_{3}, v_{1}\right)=k$. In this case

$$
\begin{aligned}
& f_{M}\left(v_{1}\right)=\left\{D\left(v_{1}, v_{1}\right), D\left(v_{1}, v_{2}\right), D\left(v_{1}, v_{3}\right)\right\} \\
& f_{M}\left(v_{2}\right)=\left\{D\left(v_{2}, v_{1}\right), D\left(v_{2}, v_{2}\right), D\left(v_{2}, v_{3}\right)\right\} \\
& f_{M}\left(v_{3}\right)=\left\{D\left(v_{3}, v_{1}\right), D\left(v_{3}, v_{2}\right), D\left(v_{3}, v_{3}\right)\right\}
\end{aligned}
$$

Since detour distance is a metric for any connected graph, thus $f_{M}\left(v_{1}\right)=$ $\{0, k\}=f_{M}\left(v_{2}\right)=f_{M}\left(v_{3}\right)$. A contradiction to the fact that $M$ is a $d d p d$-set of $G$.

Case 2: $D\left(v_{1}, v_{2}\right)=D\left(v_{2}, v_{3}\right)=k \neq D\left(v_{3}, v_{1}\right)=l$. In this case

$$
f_{M}\left(v_{1}\right)=\left\{D\left(v_{1}, v_{1}\right), D\left(v_{1}, v_{2}\right), D\left(v_{1}, v_{3}\right)\right\}
$$




$$
f_{M}\left(v_{3}\right)=\left\{D\left(v_{3}, v_{1}\right), D\left(v_{3}, v_{2}\right), D\left(v_{3}, v_{3}\right)\right\}
$$

Since detour distance is a metric for any connected graph, thus $f_{M}\left(v_{1}\right)=$ $\{0, k, l\}=f_{M}\left(v_{3}\right)$. A contradiction to the fact that $M$ is a $d d p d$-set of $G$.

In each of the cases we get a contradiction to the fact that $M$ is a $d d p d$-set of $G$. Hence the proof follows.

We now proceed to characterize the graphs admitting a $d d p d$-set $M$ of cardinality 1 , but before that we prove the following.

Lemma 18. Let $G$ be a graph admitting a ddpd-set $M$ of cardinality 1, say $M=\{x\}$, then $x$ is a pendant vertex of $G$.

Proof. Let $G$ be a graph admitting a $d d p d$-set $M$ of cardinality 1 , say $M=$ $\{x\}$. We claim that $x$ is a pendant vertex of $G$. The proof is by contradiction. Suppose that the set of neighbors of $x$, denoted by $N(x)$, contains at least two elements.

Case 1: Suppose that for all distinct pairs of elements $y, z$ in $N(x)$, there exists no $y-z$ path in $G-x$. Then clearly $f_{M}(y)=f_{M}(z)=\{1\}$, a contradiction to the fact that $M$ is a $d d p d$-set of $G$.

Case 2: Suppose that there exists two distinct elements $y, z$ in $N(x)$ such that there is a $y-z$ path in $G-x$. Then, amongst all pairs $y, z \in N(x)$, find a pair $a, b$ having an $a-b$ path $P$ of maximal length in $G-x$. Then $f_{M}(a)=f_{M}(b)=\{1+|E(P)|\}$, a contradiction to the fact that $M$ is a $d d p d$-set of $G$.

Thus, $x$ is a pendant vertex of $G$.

Theorem 19. A graph $G$ has a ddpd-set of cardinality 1 if and only if it is a path.

Proof. Let $G$ be a graph admitting a $d d p d$-set $M$ of cardinality 1, say $M=\{x\}$. In view of Lemma $18, x$ is a pendant vertex of $G$. We will now show that $G$ is a path. Assume to the contrary, then there exists a vertex in $V(G)$ of degree three or more. Let $w$ be the unique vertex of degree three or more that has the smallest distance to $x$. Thus, $G$ contains an $x-w$ path $x, x_{1}, x_{2}, \ldots, x_{k}, w$, with $w$ having at least three neighbors $x_{k}, y$ and $z$ say.

Case 1: Suppose $G$ has no cycles. Then, $f_{M}(y)=\{D(x, y)\}=\{D(x, w)+$ $D(w, y)\}$ and $f_{M}(z)=\{D(x, z)\}=\{D(x, w)+D(w, z)\}$. But $D(w, y)=$ $D(w, z)=1, G$ being a graph devoid of cycles. Hence $f_{M}(y)=\{D(x, w)+1\}=$ $f_{M}(z)$, a contradiction to the fact that $M$ is a $d d p d$ set. 
Case 2: Suppose $G$ contains a cycle.

Subcase (2.1): Suppose the degree of $w$ is exactly 3. If there exists no cycle in $G$ containing the vertices $y$ and $z$, then $f_{M}(y)=f_{M}(z)=k+2$, a contradiction. If there exists a cycle in $G$ containing the vertices $y$ and $z$, then consider the set of all $a-b$ paths in $G-w$, where $a, b$ vary over $N(w)-x_{k}$. Let $P$ denote a path of maximal length in this set, and suppose this path is from vertex $c$ to vertex $d$. Then $f_{M}(c)=f_{M}(d)$, a contradiction.

Subcase (2.2): Suppose the degree of $w$ is at least 4. If there exists no pairs of distinct vertices $a, b \in N(w)-x_{k}$ that lie in a cycle of $G$, then $f_{M}(y)=f_{M}(z)$, a contradiction. Now suppose there exist a cycle in $G$ that contains two vertices of $N(w)-x_{k}$. Amongst all pairs $a, b \in N(w)-x_{k}$, pick a pair that has the longest $a-b$ path in $G-w$. Suppose this pair is $\{c, d\}$. Then, $f_{M}(c)=f_{M}(d)$, a contradiction.

Having characterized the graphs admitting a $d d p d$-set $M$ of cardinality 1 , we now proceed to show that there exists no graphs with $d d p d$-set $M$ of cardinality 2 .

Theorem 20. There exists no ddpd-set of cardinality 2 .

Proof. Let $M=\{u, v\}$ be a $d d p d$-set of a graph $G$. Since detour distance is a metric for any connected graph, thus for any two vertices $u, v \in V$, we have $D(u, v)=D(v, u)$, which implies that $f_{M}(u)=f_{M}(v)$. A contradiction to the fact that $M$ is a $d d p d$-set of $G$.

Theorem 21. If $M$ be a ddpd-set of $G$ then, any vertex of $G$ is adjacent to at most two pendant vertices. Further, if $G$ has a vertex with exactly two pendant vertices adjacent to it then, exactly one of them belongs to $M$.

Proof. Let $M=\left\{u_{1}, u_{2}, u_{3}, \ldots, u_{k}\right\}$ be a $d d p d$-set of $G$ and $v \in V$ such that it is adjacent to at least three pendant vertices $x_{1}, x_{2}, x_{3}$. Then,

$$
f_{M}\left(x_{i}\right)=\left\{D\left(x_{i}, v_{1}\right), D\left(x_{i}, v_{2}\right), \ldots, D\left(x_{i}, v_{k}\right)\right\}, 1 \leq i \leq 3
$$

Case 1: If $x_{1}, x_{2}, x_{3} \notin M$, then for any $v_{j} \in M, 1 \leq j \leq k$, we have

$$
D\left(x_{i}, v_{j}\right)=D\left(x_{i}, v\right)+D\left(v, v_{j}\right), 1 \leq i \leq 3
$$

But $x_{1} v, x_{2} v, x_{3} v$ are bridges in $G$ and in view of Theorem $1 D\left(x_{i}, v\right)=1$, $1 \leq i \leq 3$. Hence, we have

$$
D\left(x_{i}, v_{j}\right)=1+D\left(v, v_{j}\right), 1 \leq i \leq 3
$$


By 1 and 2 we get $f_{M}\left(x_{1}\right)=f_{M}\left(x_{2}\right)=f_{M}\left(x_{3}\right)$. Which is a contradiction to the fact that $M$ is a $d d p d$-set in $G$.

Case 2: If $x_{1}, x_{2}, x_{3} \in M$. Without loss of generality let us assume that $v_{1}=x_{1}, v_{2}=x_{2}$, and $v_{3}=x_{3}$. Since $x_{1}, x_{2}, x_{3}$ are pendant vertices we have the following

$$
D\left(v_{i}, v_{j}\right)= \begin{cases}0, & \text { if } 1 \leq i, j \leq 3 \text { and } i=j ; \\ 2, & \text { if } 1 \leq i, j \leq 3 \text { and } i \neq j ; \\ 1+D\left(v, v_{j}\right), & \text { if } 1 \leq i \leq 3, i \neq j \text { and } 4 \leq j \leq k .\end{cases}
$$

By 1 and 3 we get $f_{M}\left(x_{1}\right)=f_{M}\left(x_{2}\right)=f_{M}\left(x_{3}\right)$. Which is a contradiction to the fact that $M$ is a $d d p d$-set in $G$.

Case 3: If $x_{1}, x_{2} \in M$ and $x_{3} \notin M$. Without loss of generality let us assume that $v_{1}=x_{1}, v_{2}=x_{2}$. Since $x_{1}, x_{2}$ are pendant vertices we have the following

$$
D\left(v_{i}, v_{j}\right)= \begin{cases}0, & \text { if } 1 \leq i, j \leq 2 \text { and } i=j ; \\ 2, & \text { if } 1 \leq i, j \leq 2 \text { and } i \neq j ; \\ 1+D\left(v, v_{j}\right), & \text { if } 1 \leq i \leq 2, i \neq j \text { and } 3 \leq j \leq k .\end{cases}
$$

By 1 and 4 we get $f_{M}\left(x_{1}\right)=f_{M}\left(x_{2}\right)$. Which is a contradiction to the fact that $M$ is a $d d p d$-set in $G$.

Case 4: If $x_{1}, x_{2} \notin M$ and $x_{3} \in M$. Without loss of generality let us assume that $v_{1}=x_{3}$. Since $x_{1}, x_{2}, x_{3}$ are pendant vertices we have the following

$$
D\left(v_{i}, v_{j}\right)= \begin{cases}2, & \text { if } 1 \leq i \leq 2 \text { and } j=1 ; \\ 1+D\left(v, v_{j}\right), & \text { if } 1 \leq i \leq 2, \text { and } 2 \leq j \leq k .\end{cases}
$$

By 1 and 5 we get $f_{M}\left(x_{1}\right)=f_{M}\left(x_{2}\right)$. Which is a contradiction to the fact that $M$ is a $d d p d$-set in $G$.

In each of the cases we get a contradiction to the fact that $M$ is a $d d p d$-set of $G$. Hence the proof follows.

Theorem 22. Complete bipartite graphs $K_{X, Y}$ possess a ddpd set $M$ if and only if either $|X|=|Y|=1$ or $|X|=1,|Y|=2$.

Proof. Let $K_{X, Y}$ such that either $|X|=|Y| \neq 1$ or $|X| \neq 1,|Y| \neq 2$ possess a $d d p d$-set $M$. In view of Theorem 19 and Theorem $20,|M| \geq 3$. Let $M=\left\{u_{1}, u_{2}, \ldots u_{k}\right\}$, where $k \geq 3$.

Case 1: Suppose $K_{X, Y}$ contains a hamiltonian cycle. Since $K_{X, Y}$ contains a hamiltonian cycle therefore $|X|=|Y|$.

Subcase (1.1): Let $M \subseteq X$ and $M \cap Y=\emptyset$. Let $u_{i}, u_{j} \in M$ then $f_{M}\left(u_{i}\right)=$ $\{0,2(|X|+|Y|)-2\}=f_{M}\left(u_{j}\right)$ a contradiction. 
Subcase (1.2): Let $M \cap X \neq \emptyset$ and $M \cap Y \neq \emptyset$. Let $u_{i}, u_{j} \in M \cap X$ and $v \in M \cap Y$ then $f_{M}\left(u_{i}\right)=\{0,2(|X|+|Y|)-1,2(|X|+|Y|)-2\}=f_{M}\left(u_{j}\right)$ a contradiction.

Case 2: Suppose $K_{X, Y}$ does not contain a hamiltonian cycle. Since $K_{X, Y}$ does not contain a hamiltonian cycle therefore $|X| \neq|Y|$, without loss of generality let $|X|>|Y|$.

Subcase (2.1): Let $M \subseteq X$ and $M \cap Y=\emptyset$. Let $u_{i}, u_{j} \in M$ then $f_{M}\left(u_{i}\right)=$ $\{0,2|Y|\}=f_{M}\left(u_{j}\right)$ a contradiction.

Subcase (2.2): Let $M \subseteq Y$ and $M \cap X=\emptyset$. Let $u_{i}, u_{j} \in M$ then $f_{M}\left(u_{i}\right)=$ $\{0,2|X|\}=f_{M}\left(u_{j}\right)$ a contradiction.

Subcase (2.3): Let $M \cap X \neq \emptyset$ and $M \cap Y \neq \emptyset$. Let $u_{i}, u_{j} \in M \cap X$ and $v \in M \cap Y$ then $f_{M}\left(u_{i}\right)=\{0,2|Y|, 2|Y|-1\}=f_{M}\left(u_{j}\right)$ a contradiction.

Hence if $K_{X, Y}$ is such that either $|X|=|Y| \neq 1$ or $|X| \neq 1,|Y| \neq 2$ then $K_{X, Y}$ does not possess a $d d p d$-set $M$. To see that $K_{X, Y}$ such that either $|X|=|Y|=1$ or $|X|=1,|Y|=2$ possess a $d d p d$-set $M$ note that $K_{X, Y}$ for $|X|=|Y|=1$ is $P_{2}$ and $K_{X, Y}$ for $|X|=1,|Y|=2$ is a $P_{3}$ and $P_{n}$ are known to be $d d p d$-graphs.

A beautiful class of binary trees is the class of Fibonacci trees [6], [7]. Fibonacci trees of order $n$ has $F_{n}$ terminal vertices., where $\left\{F_{n}\right\}$ are the Fibonacci numbers $F_{0}=1=F_{1}, F_{n}=F_{n-1}+F_{n-2}$, and is defined inductively as follows: If $n=1$ or 2 , the Fibonacci tree of order $n$ is simply the root only. If $n \geq 3$ the left subtree of the Fibonacci tree of order $n$ is the Fibonacci tree of order $n-1$; and the right subtree is the Fibonacci tree of order $n-2$. The Fibonacci tree of order $n$ will be denoted by $T_{n}$ for brevity.

We conclude this section by proposing the following which we strongly believe it to be true.

Conjecture 23. Fibonacci trees $T_{n}$ are ddpd-graph for all values on $n$.

\section{References}

[1] F. Harary, Graph Theory, Addison-Wesley Publishing Company, Inc., Reading, Massachusetts, 1969.

[2] Gary Chartrand, Garry L. Johns, Songlin Tian, Detour Distance in Graphs, Annals of Discrete Mathematics, 55 (1993), 127-136.

[3] K.A. Germina, Distance-patterns of vertices in a graph, International Mathematical Forum, 5, No. 34 (2010), 1697-170. 
[4] K.A. Germina, Alphy Joseph, Sona Jose, Distance neighbourhood pattern matrices, European Journal of Pure and Applied Mathematics, 3, No. 4 (2010), 748-764.

[5] K.A. Germina, Sona Jose, Distance neighbourhood pattern matrices of trees, International Mathematical Fourm, 6, No. 12 (2011), 591-604.

[6] R.P. Grimaldi, Properties of Fibonacci trees, In: Proceedings of the Twenty-Second Southeastern Conference on Combinatorics, Graph Theory, and Computing, Baton Rouge, LA, 1991, 84, 21-32.

[7] D.E. Knuth, The art of computer programming, Volume 3, Addison-Wesley Publishing Co., Reading, Mass.-London-Don Mills, Ont. (1973); Sorting and searching, Addison-Wesley Series in Computer Science and Information Processing. 